\title{
Controlled Attenuation Parameter for Quantification of Steatosis: Which Cut-Offs to Use?
}

\author{
Roxana Sirli (iD) and Ioan Sporea \\ Department of Gastroenterology and Hepatology, Advanced Regional Research Center in Gastroenterology and Hepatology, \\ "Victor Babeş" University of Medicine and Pharmacy, 156, Liviu Rebreanu Bv., Timişoara 300723, Romania \\ Correspondence should be addressed to Roxana Sirli; roxanasirli@gmail.com
}

Received 12 November 2020; Revised 13 March 2021; Accepted 17 March 2021; Published 26 March 2021

Academic Editor: Branka Filipović

Copyright (c) 2021 Roxana Sirli and Ioan Sporea. This is an open access article distributed under the Creative Commons Attribution License, which permits unrestricted use, distribution, and reproduction in any medium, provided the original work is properly cited.

\begin{abstract}
Chronic liver diseases (CLDs) are a public health problem, even if frequently they are underdiagnosed. Hepatic steatosis (HS), encountered not only in nonalcoholic fatty liver disease (NAFLD) but also in chronic viral hepatitis, alcoholic liver disease, etc., plays an important role in fibrosis progression, regardless of CLD etiology; thus, detection and quantification of HS are imperative. Controlled attenuation parameter (CAP) feature, implemented in the FibroScan ${ }^{\circledR}$ device, measures the attenuation of the US beam as it passes through the liver. It is a noninvasive technique, feasible and well accepted by patients, with lower costs than other diagnostic techniques, with acceptable accuracy for HS quantification. Multiple studies have been published regarding CAP performance to quantify steatosis, but due to the heterogeneity of CLD etiologies, of steatosis prevalence, etc., it had widely variable calculated cut-off values, which in turn limited the day-to-day utility of CAP measurements in clinical practice. This paper reviews published studies trying to suggest cut-off values usable in clinical practice.
\end{abstract}

\section{Introduction}

Chronic liver diseases (CLDs) are a public health problem, even if frequently they are underdiagnosed. A study from 2014 estimated that 844 million individuals are affected by CLD, with a mortality rate of 2 million per year [1]. The most frequent CLDs are chronic viral hepatitis, alcoholic liver disease (ALD), and nonalcoholic fatty liver disease (NAFLD) with its progressive variant-nonalcoholic steatohepatitis (NASH). Even if effective treatments are available for chronic viral hepatitis, in NAFLD and NASH this is not the case, an alarming fact considering that the world-vide pooled prevalence of NAFLD is estimated to be $25.24 \%$ [2], ranging from approximately $13 \%$ in Africa to approximately $30 \%$ in Asia and South America. Furthermore, the prevalence of NAFLD is expected to increase since the prevalence of its etiologic factors (obesity, diabetes mellitus, hypertriglyceridemia) is increasing.

Hepatic steatosis (HS) is encountered not only in NAFLD, but also in chronic viral hepatitis, alcoholic liver disease, etc. Several studies demonstrated that HS plays an important role in fibrosis progression, regardless of CLD etiology $[3,4]$, and that it impairs response to treatment in chronic viral hepatitis [5].

\section{Diagnosis of Hepatic Steatosis}

Considering all these facts, detection and quantification of HS are imperative, but also a challenge. Detection of HS relies mainly on imaging methods. B-mode ultrasonography is usually the first-line imaging method to detect HS, but it cannot assess the presence of inflammation and it is imprecise to assess steatosis severity, especially mild [6, 7]. Magnetic resonance imaging (MRI) techniques, especially proton density fat fraction (PDFF), are very accurate to detect and quantify HS [8], but they are very expensive and not available enough to be used for assessment of such a large number of patients.

Liver biopsy is considered the gold standard for assessing HS severity, as well as inflammation and fibrosis, when they are present $[6,7]$. According to histologic findings, liver steatosis is 
classified as absent- $S_{0}$ (normal liver), when less than $5 \%$ of the hepatocytes have fatty infiltration; mild- $S_{1}$, when 5 up to $33 \%$ of the hepatocytes present fatty infiltration; moderate- $S_{2}, 33-66 \%$ of the hepatocytes with fatty infiltration; and severe- $S_{3}$, more than $66 \%$ of the hepatocytes with fatty infiltration [6, 7]. However, liver biopsy is an invasive method, poorly accepted by the patients, especially if repetitive, and there are some problems regarding inter-observer variability in assessing the sample, as well as regarding sampling errors [9]. Furthermore, the applicability of liver biopsy to assess such a huge number of patients is highly questionable.

Considering all these facts, noninvasive methods have been developed to assess HS, as well as inflammation and fibrosis (when present). They include biomarkers and imaging techniques $[6,7]$. Among the imaging techniques, the controlled attenuation parameter (CAP) feature, implemented on the FibroScan ${ }^{\circledR}$ device, seems the most promising noninvasive test to quantify HS.

\section{Controlled Attenuation Parameter (CAP): Technical Data}

Vibration-controlled transient elastography (VCTE) (FibroScan ${ }^{\circledR}$, EchoSens, Paris, France) is an ultrasound-based elastography technique developed more than 15 years ago, firstly used for fibrosis assessment in chronic liver diseases. It is the most validated elastography technique, accepted by international guidelines as a reliable tool to quantify liver fibrosis $[10,11]$. VCTE measures the velocity of shear waves generated inside the liver by a mechanical impulse. In CLD, liver stiffness increases with the progression of fibrosis. The stiffer the liver is, the higher the shear waves' velocity.

Several years later, CAP feature was added to the FibroScan ${ }^{\circledR}$ device. It measures the attenuation of the US beam as it passes through the liver. CAP correlates with the viscoelastic characteristics of the liver, dependent in their turn on the quantity of fat droplets in the hepatocytes [12]. CAP measurements can be performed by either the M or XL probes (chosen according to the skin to liver capsule distance), and the results are expressed in decibels per meter $(\mathrm{dB} / \mathrm{m})$, ranging from 100 to $400 \mathrm{~dB} / \mathrm{m}$ [13]. At the beginning, CAP was available only on the M probe of the FibroScan ${ }^{\circledR}$. Later, it was implemented also on the XL probe developed for obese subjects.

The initial studies regarding CAP showed excellent feasibility-92.3\% of cases with only the M probe [13], improved to $96.8 \%$ when both $\mathrm{M}$ and XL probes have been used [14], also with excellent reproducibility, inter-rater agreement $0.82-0.84$ with the $M$ probe $[15,16]$, but lower with the XL probe, 0.75 and 0.65 , respectively $[14,15]$.

No quality technical parameters have been recommended by the producers to ensure reliable measurements. Therefore, most authors used the quality criteria recommended for VCTE: 10 valid measurements with an IQR/ $\mathrm{M}<30 \%$ [17, 18]. A study published in 2017 recommended as a quality criterion for CAP measurements an IQR $<40 \mathrm{~dB} /$ $m$ [19]. When this quality criterion was used, the AUROC of CAP to assess steatosis as compared to liver biopsy increased from 0.77 to 0.9 . Another study has set the IQR upper limit at $30 \mathrm{~dB} / \mathrm{m}$ [8], while another study found no difference in CAP performance when the IQR was $\geq 30 \mathrm{~dB} / \mathrm{m}$ or $\geq 40 \mathrm{~dB} / \mathrm{m}$ [20]. A recently published study demonstrated that CAP$\mathrm{IQR} / \mathrm{M}<0.3$ as a quality criterion improves accuracy and feasibility of CAP measurements, performing better than the IQR $<40 \mathrm{~dB} / \mathrm{m}$ criterion [21].

Regarding the use of $\mathrm{M}$ vs. XL probe to assess steatosis grade by CAP, data is still conflicting. In a study performed in a Caucasian population, the cut-off and performance were similar for $M$ vs. XL probe [22], while in a smaller study performed in a Chinese population, cut-off values were higher with the XL probe, but the performance was similar [23]. In a very recent study in Japanese population, cut-off values were higher for the XL probe, but there were no significant differences in accuracy [24].

Several studies demonstrated that CAP measurements are not influenced by the severity of liver fibrosis, nor by the presence of cirrhosis [25-28]. However, several factors have been proven to influence CAP values, among them BMI $[29,30]$, the presence of diabetes and etiology, especially NAFLD [29], while CAP values higher than $300 \mathrm{~dB} / \mathrm{m}$ may lead to an overestimation of fibrosis severity by VCTE in patients with lower stages of fibrosis [31].

\section{Controlled Attenuation Parameter (CAP): Predictive Value for Steatosis Severity in Individual Studies}

Up to date, numerous studies have been published regarding the predictive value of CAP for steatosis severity. We summarized in Table 1 data from studies including more than 100 subjects, with liver biopsy as the reference method, CAP measurements being performed with the $M$ probe (Table 1).

As it can be seen, the performance of CAP for detecting any steatosis $(S \geq 1)$ is very good, the AUROC usually being higher than 0.8 . In populations with mixed etiology of CLD, the AUROCs remain also high for diagnosing more severe steatosis $\left(S_{2}\right.$ and $\left.S_{3}\right)$. However, in NAFLD population, the AUROCs for diagnosing moderate $\left(S_{2}\right)$ and severe $\left(S_{3}\right)$ steatosis decrease, sometimes as low as 0.58 [39], or even 0.37 [38]. Nevertheless, the severity of fat infiltration in NAFLD does not affect prognosis [45], so the important thing is to detect even mild steatosis $\left(S_{1}\right)$, for which CAP is much better than B-mode ultrasonography [46].

The largest individual study assessing the value of CAP for predicting fibrosis severity was published in 2019 by Eddowes et al. [20]. It was a multicenter prospective study that included 450 patients with NAFLD evaluated by CAP/TE and liver biopsy. The AUROCs of CAP to identify patients' steatosis were as follows: for $S \geq S_{1}$-AUROC of 0.87 ; for $S \geq S_{2}-0.77$; while for $S_{3}$ it was 0.70 . Youden cut-off values were $302 \mathrm{~dB} / \mathrm{m}$ for $S \geq S_{1}, 331 \mathrm{~dB} / \mathrm{m}$ for $S \geq S_{2}$, and $337 \mathrm{~dB} / \mathrm{m}$ for $S_{3}$.

The cut-offs also vary a lot among the studies. An explanation could be the relatively small number of patients included in each study, the heterogeneity among groups regarding etiology, overall steatosis prevalence, and also among steatosis severity groups. 
TABLE 1: Performance of CAP (M probe) to diagnose steatosis in patients with CLD, with liver biopsy as the reference method.

\begin{tabular}{|c|c|c|c|c|c|c|c|c|c|}
\hline \multirow[b]{2}{*}{ Author } & \multirow[b]{2}{*}{$\begin{array}{c}\text { No. of } \\
\text { patients }\end{array}$} & \multirow[b]{2}{*}{ Etiology } & \multirow[b]{2}{*}{$\begin{array}{c}\text { Prevalence of } \\
S \geq 1(\%)\end{array}$} & \multicolumn{2}{|c|}{$S \geq 1$} & \multicolumn{2}{|c|}{$S \geq 2$} & \multicolumn{2}{|c|}{$S=3$} \\
\hline & & & & $\begin{array}{l}\text { Cut-off (dB/ } \\
\mathrm{m})\end{array}$ & AUROC & $\begin{array}{l}\text { Cut-off } \\
(\mathrm{dB} / \mathrm{m})\end{array}$ & AUROC & $\begin{array}{l}\text { Cut-off } \\
(\mathrm{dB} / \mathrm{m})\end{array}$ & AUROC \\
\hline Sasso [28] & 615 & $\mathrm{HCV}$ & 30 & 222 & 0.80 & 233 & 0.86 & 290 & 0.88 \\
\hline Myers [27] & 153 & Mixed & 65 & 283 & 0.81 & - & - & - & - \\
\hline $\begin{array}{l}\text { De Ledinghen } \\
\text { [25] }\end{array}$ & 112 & Mixed & 48 & 215 & 0.84 & 252 & 0.86 & 296 & 0.93 \\
\hline Chan [32] & 105 & NAFLD & 97 & 263 & 0.97 & 281 & 0.86 & 283 & 0.75 \\
\hline $\begin{array}{l}\text { De Ledinghen } \\
\text { [13] }\end{array}$ & 440 & Mixed & 51.5 & - & 0.79 & - & 0.84 & - & 0.84 \\
\hline Ferraioli [26] & 114 & Mixed & 42.6 & 219 & 0.76 & 296 & 0.82 & - & - \\
\hline $\begin{array}{l}\text { Lupsor-Platon } \\
\text { [33] }\end{array}$ & 201 & Mixed & 45.3 & 260 & 0.81 & 285 & 0.82 & 194 & 0.84 \\
\hline Shen [34] & 332 & Mixed & 42.5 & 255 & 0.88 & 283.5 & 0.90 & 293.5 & 0.84 \\
\hline $\begin{array}{l}\text { De Ledinghen } \\
\text { [35] }\end{array}$ & 261 & NAFLD & 100 & - & - & 310 & 0.80 & 311 & 0.66 \\
\hline Imajo [36] & $\begin{array}{c}142(10 \\
\text { controls) }\end{array}$ & NAFLD & 83 & 236 & 0.88 & 279 & 0.73 & 302 & 0.70 \\
\hline Park [37] & 104 & NAFLD & 91 & 261 & 0.85 & 305 & 0.70 & 312 & 0.73 \\
\hline Naveau [38] & 123 & NAFLD & 81 & 298 & 0.81 & 303 & 0.58 & 326 & 0.37 \\
\hline Siddiqui [39] & 393 & NAFLD & 95 & 285 & 0.76 & 311 & 0.70 & 306 & 0.58 \\
\hline Thiele [40] & 269 & $\begin{array}{c}\text { Alcoholic liver } \\
\text { disease }\end{array}$ & 72 & $\begin{array}{l}290 \text {-rule-in } \\
220 \text {-rule-out }\end{array}$ & 0.77 & $\begin{array}{l}\text { 328-rule- } \\
\text { in } \\
257 \text {-rule- } \\
\text { out }\end{array}$ & 0.78 & $\begin{array}{l}\text { 339-rule- } \\
\text { in } \\
286 \text {-rule- } \\
\text { out }\end{array}$ & 0.83 \\
\hline Shalimar [30] & 219 & NAFLD & 93.2 & 285 & 0.96 & 331 & 0.71 & 348 & 0.75 \\
\hline Oeda [24] & 137 & NAFLD & 96.3 & - & - & 264 & 0.64 & 289 & 0.69 \\
\hline Somda [41] & 249 & Severely obese & 84.3 & 255 & 0.86 & 288 & 0.83 & 297 & 0.79 \\
\hline Eddowes [20] & 450 & NAFLD & 88 & 302 & 0.87 & 331 & 0.77 & 337 & 0.70 \\
\hline Baumeler [42] & 224 & Mixed & 62.1 & 258.5 & 0.78 & 282.5 & 0.83 & 307.5 & 0.82 \\
\hline Trowell [43] & 217 & Mixed & 43 & 278 & 0.82 & 301 & 0.79 & - & - \\
\hline Zeng [44] & 173 & Liver donors & - & 244 & 0.88 & - & 0.89 & - & - \\
\hline
\end{tabular}

CAP: controlled attenuation parameter; S: steatosis; AUROC: area under the receiver operating characteristic curve; HCV: hepatitis C virus; NAFLD: nonalcoholic fatty liver disease.

To overcome these shortcomings, meta-analyses have been performed.

\section{Controlled Attenuation Parameter (CAP): Predictive Value for Steatosis Severity in Meta-Analyses}

The first published meta-analysis included nine studies with 11 cohorts, totalizing 1771 patients with CLD of diverse etiologies [47]. The summary sensitivities and specificities values were 0.78 and 0.79 for $S \geq 1 ; 0.85$ and 0.79 for $S \geq 2$; 0.83 and 0.79 for $S_{3}$, respectively. The HSROCs were 0.85 for $S \geq 1,0.88$ for $S \geq 2$, and 0.87 for $S_{3}$. The median optimal cutoff values of CAP for $S \geq 1, S \geq 2$, and $S_{3}$ were $232.5 \mathrm{~dB} / \mathrm{m}$ (range $214-289 \mathrm{~dB} / \mathrm{m}$ ), $255 \mathrm{~dB} / \mathrm{m}$ (range $233-311 \mathrm{~dB} / \mathrm{m}$ ), and $290 \mathrm{~dB} / \mathrm{m}$ (range $266-318 \mathrm{~dB} / \mathrm{m}$ ).

The second meta-analysis included 11 studies with 13 cohorts, all of them with high methodological quality, totalizing 2076 patients with CLD of diverse etiologies [48]. The summary sensitivity, specificity, and AUC for $S \geq 1$ were $0.78,0.79$, and 0.86 , respectively; for $S \geq 2$, they were 0.82 , 0.79 , and 0.88 , respectively, while for $S_{3}$ they were $0.86,0.89$, and 0.94 , respectively. Significant heterogeneity was found among the studies for $S \geq 1$ and $S_{3}$. CAP cut-of values for $S \geq 1$ ranged from 214 to $289 \mathrm{~dB} / \mathrm{m}$, median $238 \mathrm{~dB} / \mathrm{m}$; for $S \geq 2$ they ranged from 230 to $311 \mathrm{~dB} / \mathrm{m}$, median $259 \mathrm{~dB} / \mathrm{m}$, while for $S_{3}$ CAP values ranged from 266 to $327 \mathrm{~dB} / \mathrm{m}$, median $290 \mathrm{~dB} / \mathrm{m}$.

Both meta-analyses above were not able to provide optimized cut-offs with high predictive values due to the limitations of conventional meta-analyses and to the heterogeneity of the included studies, so that a third meta-analysis was performed, this time using individual patient data from 19 studies, including 2735 CLD cases of various etiology, with liver biopsy and CAP measurements [29]. The overall performance of CAP in this meta-analysis was as follows: for $S \geq 1$ the calculated cutoff was $248 \mathrm{~dB} / \mathrm{m}$, with 0.68 sensitivity and 0.82 specificity (AUROC 0.82); for $S \geq 2$, the calculated cut-off was $268 \mathrm{~dB} / \mathrm{m}$, with 0.77 sensitivity and 0.81 specificity (AUROC 0.86 ), while for $S_{3}$ the calculated cut-off was $280 \mathrm{~dB} / \mathrm{m}$, with 0.88 sensitivity and 0.77 specificity (AUROC 0.88 ).

Another important finding of this last meta-analysis is the fact that, among etiologies, only NAFLD seems to influence CAP values. In other words, NAFLD patients have higher CAP values (by $10 \mathrm{~dB} / \mathrm{m}$ ) as compared with all other etiologies of CLD for the same grade of histologic steatosis [29]. Furthermore, it was calculated that BMI, as well as the 
TABLE 2: Main advantages and weaknesses of CAP/VCTE.

\begin{tabular}{|c|c|}
\hline Advantages & Weaknesses \\
\hline (i) Reproducible method & (i) Expensive equipment \\
\hline $\begin{array}{l}\text { (ii) Well accepted by the patients and thus repeatable } \\
\text { assessment possible for follow-up }\end{array}$ & (ii) Not feasible in patients with ascites \\
\hline $\begin{array}{l}\text { (iii) Good results for noninvasive steatosis assessment in } \\
\text { patients with CLD, including NASH }\end{array}$ & $\begin{array}{l}\text { (iii) Increased number of unreliable measurements in patients with high } \\
\text { BMI, especially with } M \text { probe }\end{array}$ \\
\hline $\begin{array}{l}\text { (iv) CAP could be used as a screening tool in patients at risk for } \\
\text { NAFLD/NASH }\end{array}$ & (iv) CAP not very accurate to differentiate $S \geq 2$ from $S_{3}$ \\
\hline $\begin{array}{l}\text { (v) Real-time assessment not only of steatosis but also of fibrosis } \\
\text { severity }\end{array}$ & $\begin{array}{l}\text { (v) TE not very accurate to differentiate patients without fibrosis and } \\
\text { those with mild fibrosis and patients with moderate vs. mild fibrosis }\end{array}$ \\
\hline $\begin{array}{l}\text { (vi) Reliable tool for noninvasive assessment of fibrosis, } \\
\text { recognized by international guidelines } \\
\text { (vii) Results and technical parameters IQR/M available in real } \\
\text { time, automatically calculated by the device's software }\end{array}$ & - \\
\hline
\end{tabular}

CAP: controlled attenuation parameter; VCTE: vibration-controlled transient elastography.

presence of diabetes mellitus, influences CAP values. Considering these findings, the authors propose an algorithm to correct the measured CAP values, and to apply the cut-offs only after the corrections are made. These correction include deducting $10 \mathrm{~dB} / \mathrm{m}$ for the presence of NAFLD/ $\mathrm{NASH}$, as well as for diabetes mellitus, deducting $4.4 \mathrm{~dB} / \mathrm{m}$ for each BMI unit over $25 \mathrm{~kg} / \mathrm{m}^{2}$, or adding $4.4 \mathrm{~dB} / \mathrm{m}$ for each BMI unit bellow $25 \mathrm{~kg} / \mathrm{m}^{2}$.

Finally, a recently published meta-analysis assessed only NAFLD patients (1297 subjects) evaluated by liver biopsy and CAP in nine studies [49]. The mean AUROC, pooled sensitivity, and pooled specificity for diagnosing $S \geq 1$ were 0.96 , 0.87 , and 0.91 , respectively; for $S \geq 2$, they were $0.82,0.85$, and 0.74 , respectively, while for $S_{3}$ they were $0.70,0.76$, and 0.58 , respectively. As observed in individual studies (Table 1), in NAFLD patients the performance of CAP to diagnose steatosis severity decreases as the steatosis progresses. No polled cut-off values have been calculated in this meta-analysis.

\section{Controlled Attenuation Parameter, Transient Elastography, and NAFLD/NASH}

As mentioned before, the prevalence of NAFLD/NASH is increasing worldwide and in the future will be the main cause of liver-related morbidity and mortality. Considering the high number of patients and the fact that not all patients with NAFLD will develop NASH and liver related events, it is not feasible to try to evaluate all of them by liver biopsy, thus the utility of noninvasive methods. As shown before, individual studies [20, 24, 30, 32, 35-39] and meta-analyses [49] proved the value of CAP for diagnosing steatosis in patients with NAFLD/NASH, even if accuracy decreases with the severity of steatosis [49].

VCTE is the most validated elastographic method for fibrosis assessment in NAFLD/NASH. The cut-off values for different stages of fibrosis vary according to the probe used. For the XL probe (developed especially for obese patients), the cut-offs are as follows: $6.2 \mathrm{kPa}$ for $F \geq 2,7.2 \mathrm{kPa}$ for $F \geq 3$, and $7.9 \mathrm{kPa}$ for $F_{4}$ [50]. For the $\mathrm{M}$ probe the cut-offs are as follows: $7 \mathrm{kPa}$ for $F \geq 2,8.7 \mathrm{kPa}$ for $F \geq 3$, and $10.3 \mathrm{kPa}$ for $F_{4}$ [51]. In a recent meta-analysis that included 854 NAFLD patients from eight studies, TE had 79\% Se and 75\% Sp for diagnosing $F \geq 2$ and $85 \%$ Se and $\mathrm{Sp}$ for diagnosing $F \geq 3$, while for cirrhosis the Se and Sp were 92\% [52]. No cut-offs were provided. The accuracy of TE increases with the severity of fibrosis; thus, TE is a very good method to rule in and to rule out cirrhosis.

\section{Final Considerations}

The ideal diagnostic test should be accurate, available, noninvasive, feasible, inexpensive, and acceptable by the patient. All the data that we presented above suggest that CAP is a feasible test with good accuracy for the detection and quantification of hepatic steatosis, if clinical aspects, such as $\mathrm{BMI}$ and presence of diabetes mellitus and of NAFLD/NASH, are taken into consideration. Regarding availability, FibroScan ${ }^{\circledR}$ device is readily available in European countries such as France and even Romania, and, a few years ago, FDA accepted it as a valuable tool to assess fibrosis in the United States. Since it is noninvasive, and it takes only a few minutes to perform, VCTE and CAP are well accepted by the patients. Thus, in some countries, VCTE and serologic markers replaced almost entirely liver biopsy for fibrosis severity assessment [53]. Regarding CAP costs, they are included in those of VCTE assessment of fibrosis and are much lower than of PDFF-MRI, even if with a small loss of accuracy.

Considering all of the above, the rise in NAFLD/NASH prevalence, as well as the steatosis impact on the prognosis of CLD, CAP could be used as a screening tool in patients at risk for NAFLD/NASH (diabetics, obese, patients with metabolic syndrome). Regarding cut-offs to be used, those calculated by the Karlas meta-analysis seem the most robust since they were calculated starting from a large individual data-base meta-analysis and since they take into consideration factors known to influence CAP measurements [29].

The main advantages and weaknesses of CAP/VCTE are summarized in Table 2.

\section{Conclusion}

Controlled attenuation parameter is a valuable tool to detect hepatic steatosis in day-to-day clinical practice. Cut-off values of $248 \mathrm{~dB} / \mathrm{m}, 268 \mathrm{~dB} / \mathrm{m}$, and $280 \mathrm{~dB} / \mathrm{m}$, corrected by 
BMI and presence of co-morbidities, can be taken into consideration to diagnose $S \geq 1, S \geq 2$, and $S_{3}$.

\section{Conflicts of Interest}

The authors declare that there are no conflicts of interest regarding the publication of this paper.

\section{Acknowledgments}

Part of the research published in this paper was made with support from the grant awarded by the "Victor Babeş" University of Medicine and Pharmacy Timişoara-Acronim: BRLIV, under Contract 2EXP/2020.

\section{References}

[1] P. Byass, "The global burden of liver disease: a challenge for methods and for public health," BMC Medicine, vol. 12, p. 159, 2014.

[2] S. Mitra, A. De, and A. Chowdhury, "Epidemiology of nonalcoholic and alcoholic fatty liver diseases," Translational Gastroenterology and Hepatology, vol. 5, p. 16, 2020.

[3] S. Singh, A. M. Allen, Z. Wang, L. J. Prokop, M. H. Murad, and R. Loomba, "Fibrosis progression in nonalcoholic fatty liver vs. nonalcoholic steatohepatitis: a systematic review and meta-analysis of paired-biopsy studies," Clinical Gastroenterology and Hepatology, vol. 13, no. 4, pp. 643-654, 2015.

[4] H. K. Dyal, M. Aguilar, T. Bhuket et al., "Concurrent obesity, diabetes, and steatosis increase risk of advanced fibrosis among HCV patients: a systematic review," Digestive Diseases and Sciences, vol. 60, no. 9, pp. 2813-2824, 2015.

[5] A. S. F. Lok, J. E. Everhart, R. T. Chung et al., "Hepatic steatosis in hepatitis $\mathrm{C}$ : comparison of diabetic and nondiabetic patients in the hepatitis $\mathrm{C}$ antiviral long-term treatment against cirrhosis trial," Clinical Gastroenterology and Hepatology, vol. 5, no. 2, pp. 245-254, 2007.

[6] European Association for the Study of the Liver, European Association for the Study of Diabetes, and European Association for the Study of Obesity, "EASL-EASD-EASO clinical practice guidelines for the management of non-alcoholic fatty liver disease," Journal of Hepatology, vol. 64, no. 6, pp. 1388-1402, 2016.

[7] N. Chalasani, Z. Younossi, J. E. Lavine et al., "The diagnosis and management of nonalcoholic fatty liver disease: practice guidance from the American association for the study of liver diseases," Hepatology, vol. 67, no. 1, pp. 328-357, 2018.

[8] C. Caussy, M. H. Alquiraish, P. Nguyen et al., "Optimal threshold of controlled attenuation parameter with MRIPDFF as the gold standard for the detection of hepatic steatosis," Hepatology, vol. 67, no. 4, pp. 1348-1359, 2018.

[9] P. Bedossa and F. Carrat, "Liver biopsy: the best, not the gold standard," Journal of Hepatology, vol. 50, no. 1, pp. 1-3, 2009.

[10] European Association for Study of Liver and Asociacion Latinoamericana para el Estudio del Higado, "EASL-ALEH Clinical Practice Guidelines: non-invasive tests for evaluation of liver disease severity and prognosis," Journal of Hepatology, vol. 63, no. 1, pp. 237-264, 2015.

[11] J. K. Lim, S. L. Flamm, S. Singh et al., "American gastroenterological association institute guideline on the role of elastography in the evaluation of liver fibrosis," Gastroenterology, vol. 152, no. 6, pp. 1536-1543, 2017.
[12] M. Sasso, M. Beaugrand, V. de Ledinghen et al., "Controlled attenuation parameter (CAP): a novel $\mathrm{VCTE}^{\mathrm{TM}}$ guided ultrasonic attenuation measurement for the evaluation of hepatic steatosis: preliminary study and validation in a cohort of patients with chronic liver disease from various causes," Ultrasound in Medicine \& Biology, vol. 36, no. 11, pp. 1825$1835,2010$.

[13] V. de Lédinghen, J. Vergniol, M. Capdepont et al., "Controlled attenuation parameter (CAP) for the diagnosis of steatosis: a prospective study of 5323 examinations," Journal of Hepatology, vol. 60, no. 5, pp. 1026-1031, 2014.

[14] R. Vuppalanchi, M. S. Siddiqui, M. L. Van Natta et al., "Performance characteristics of vibration-controlled transient elastography for evaluation of nonalcoholic fatty liver disease," Hepatology, vol. 67, no. 1, pp. 134-144, 2018.

[15] G. Ferraioli, C. Tinelli, R. Lissandrin et al., "Interobserver reproducibility of the controlled attenuation parameter (CAP) for quantifying liver steatosis," Hepatology International, vol. 8, no. 4, pp. 576-581, 2014.

[16] E. Recio, C. Cifuentes, J. Macías et al., "Interobserver concordance in controlled attenuation parameter measurement, a novel tool for the assessment of hepatic steatosis on the basis of transient elastography," European Journal of Gastroenterology \& Hepatology, vol. 25, no. 8, pp. 905-911, 2013.

[17] G. Ferraioli, V. W.-S. Wong, L. Castera et al., "Liver ultrasound elastography: an update to the world federation for ultrasound in medicine and biology guidelines and recommendations," Ultrasound in Medicine \& Biology, vol. 44, no. 12, pp. 2419-2440, 2018.

[18] C. Dietrich, J. Bamber, A. Berzigotti et al., "EFSUMB guidelines and recommendations on the clinical use of liver ultrasound elastography, update 2017 (long version)," European Journal of Ultrasound, vol. 38, no. 4, pp. e16-e47, 2017.

[19] V. W.-S. Wong, S. Petta, J.-B. Hiriart et al., "Validity criteria for the diagnosis of fatty liver by $\mathrm{M}$ probe-based controlled attenuation parameter," Journal of Hepatology, vol. 67, no. 3, pp. 577-584, 2017.

[20] P. J. Eddowes, M. Sasso, M. Allison et al., "Accuracy of FibroScan controlled attenuation parameter and liver stiffness measurement in assessing steatosis and fibrosis in patients with nonalcoholic fatty liver disease," Gastroenterology, vol. 156, no. 6, pp. 1717-1730, 2019.

[21] G. Semmler, K. Wöran, B. Scheiner et al., "Novel reliability criteria for controlled attenuation parameter assessments for non-invasive evaluation of hepatic steatosis," United European Gastroenterology Journal, vol. 8, no. 3, pp. 321-331, 2020.

[22] V. de Lédinghen, J.-B. Hiriart, J. Vergniol, W. Merrouche, P. Bedossa, and V. Paradis, "Controlled attenuation parameter (CAP) with the XL probe of the fibroscan ${ }^{\circledR}$ : a comparative study with the M probe and liver biopsy," Digestive Diseases and Sciences, vol. 62, no. 9, pp. 2569-2577, 2017.

[23] W. K. Chan, N. R. Nik Mustapha, G. L. H. Wong, V. W. S. Wong, and S. Mahadeva, "Controlled attenuation parameter using the fibroscan ${ }^{\circledR}$ XL probe for quantification of hepatic steatosis for non-alcoholic fatty liver disease in an Asian population," United European Gastroenterology Journal, vol. 5, no. 1, pp. 76-85, 2017.

[24] S. Oeda, H. Takahashi, K. Imajo et al., "Accuracy of liver stiffness measurement and controlled attenuation parameter using FibroScan ${ }^{\circledR} \mathrm{M} / \mathrm{XL}$ probes to diagnose liver fibrosis and steatosis in patients with nonalcoholic fatty liver disease: a multicenter prospective study," Journal of Gastroenterology, vol. 55, no. 4, pp. 428-440, 2020. 
[25] V. Lédinghen, J. Vergniol, J. Foucher, W. Merrouche, and B. Bail, "Non-invasive diagnosis of liver steatosis using controlled attenuation parameter (CAP) and transient elastography," Liver International, vol. 32, no. 6, pp. 911-918, 2012.

[26] G. Ferraioli, C. Tinelli, R. Lissandrin et al., "Controlled attenuation parameter for evaluating liver steatosis in chronic viral hepatitis," World Journal of Gastroenterology, vol. 20, no. 21, pp. 6626-6631, 2014.

[27] R. P. Myers, A. Pollett, R. Kirsch et al., "Controlled attenuation parameter (CAP): a noninvasive method for the detection of hepatic steatosis based on transient elastography," Liver International, vol. 32, no. 6, pp. 902-910, 2012.

[28] M. Sasso, I. Tengher-Barna, M. Ziol et al., "Novel controlled attenuation parameter for noninvasive assessment of steatosis using FibroScan ${ }^{\circledR}$ : validation in chronic hepatitis C," Journal of Viral Hepatitis, vol. 19, no. 4, pp. 244-253, 2012.

[29] T. Karlas, D. Petroff, M. Sasso et al., "Individual patient data meta-analysis of controlled attenuation parameter (CAP) technology for assessing steatosis," Journal of Hepatology, vol. 66, no. 5, pp. 1022-1030, 2017.

[30] K. R. Shalimar, R. Kumar, G. Rout et al., "Body mass index-based controlled attenuation parameter cut-offs for assessment of hepatic steatosis in non-alcoholic fatty liver disease," Indian Journal of Gastroenterology, vol. 39, no. 1, pp. 32-41, 2020.

[31] S. Petta, V. W.-S. Wong, C. Cammà et al., "Improved noninvasive prediction of liver fibrosis by liver stiffness measurement in patients with nonalcoholic fatty liver disease accounting for controlled attenuation parameter values," Hepatology, vol. 65, no. 4, pp. 1145-1155, 2017.

[32] W.-K. Chan, N. R. Nik Mustapha, and S. Mahadeva, "Controlled attenuation parameter for the detection and quantification of hepatic steatosis in nonalcoholic fatty liver disease," Journal of Gastroenterology and Hepatology, vol. 29, no. 7, pp. 1470-1476, 2014.

[33] M. Lupsor-Platon, D. Feier, H. Stefanescu et al., "Diagnostic accuracy of controlled attenuation parameter measured by transient elastography for the non-invasive assessment of liver steatosis: a prospective study," Journal of Gastrointestinal and Liver Disease, vol. 24, no. 1, pp. 35-42, 2015.

[34] F. Shen, R. D. Zheng, J. P. Shi et al., "Impact of skin capsular distance on the performance of controlled attenuation parameter in patients with chronic liver disease," Liver International, vol. 35, no. 11, pp. 2392-2400, 2015.

[35] V. d. Lédinghen, G. L.-H. Wong, J. Vergniol et al., "Controlled attenuation parameter for the diagnosis of steatosis in nonalcoholic fatty liver disease," Journal of Gastroenterology and Hepatology, vol. 31, no. 4, pp. 848-855, 2016.

[36] K. Imajo, T. Kessoku, Y. Honda et al., "Magnetic resonance imaging more accurately classifies steatosis and fibrosis in patients with nonalcoholic fatty liver disease than transient elastography," Gastroenterology, vol. 150, no. 3, pp. 626-637, 2016.

[37] C. C. Park, P. Nguyen, C. Hernandez et al., "Magnetic resonance elastography vs. transient elastography in detection of fibrosis and noninvasive measurement of steatosis in patients with biopsy-proven nonalcoholic fatty liver disease," Gastroenterology, vol. 152, no. 3, pp. 598-607, 2017.

[38] S. Naveau, C. S. Voican, A. Lebrun et al., "Controlled attenuation parameter for diagnosing steatosis in bariatric surgery candidates with suspected nonalcoholic fatty liver disease," European Journal of Gastroenterology \& Hepatology, vol. 29, no. 9, pp. 1022-1030, 2017.
[39] M. S. Siddiqui, R. Vuppalanchi, M. L. Van Natta et al., "Vibration-controlled transient elastography to assess fibrosis and steatosis in patients with nonalcoholic fatty liver disease," Clinical Gastroenterology and Hepatology, vol. 17, no. 1, pp. 156-163, 2019.

[40] M. Thiele, V. Rausch, G. Fluhr et al., "Controlled attenuation parameter and alcoholic hepatic steatosis: diagnostic accuracy and role of alcohol detoxification," Journal of Hepatology, vol. 68 , no. 5, pp. 1025-1032, 2018.

[41] S. Somda, A. Lebrun, H. Tranchart et al., "Adaptation of controlled attenuation parameter (CAP) measurement depth in morbidly obese patients addressed for bariatric surgery," PLoS One, vol. 14, no. 5, Article ID e0217093, 2019.

[42] S. Baumeler, W. Jochum, J. Neuweiler, I. Bergamin, and D. Semela, "Controlled attenuation parameter for the assessment of liver steatosis in comparison with liver histology: a single-centre real life experience," Swiss Medical Weekly, vol. 149, Article ID w20077, 2019.

[43] J. Trowell, J. Alukal, T. Zhang et al., "How good are controlled attenuation parameter scores from fibroscan to assess steatosis, NASH, and fibrosis?” Digestive Disease Science, 2020.

[44] Q. Zeng, Z. Song, Y. Zhao et al., "Controlled attenuation parameter by vibration-controlled transient elastography for steatosis assessment in members of the public undergoing regular health checkups with reference to magnetic resonance imaging-based proton density fat fraction," Hepatology Research, vol. 50, no. 5, pp. 578-587, 2020.

[45] P. Angulo, D. E. Kleiner, S. Dam-Larsen et al., "Liver fibrosis, but no other histologic features, is associated with long-term outcomes of patients with nonalcoholic fatty liver disease," Gastroenterology, vol. 149, no. 2, pp. 389-397, 2015.

[46] G. Ferraioli, C. Tinelli, A. De Silvestri et al., "The clinical value of controlled attenuation parameter for the noninvasive assessment of liver steatosis," Liver International, vol. 36, no. 12, pp. 1860-1866, 2016.

[47] K.-Q. Shi, J.-Z. Tang, X.-L. Zhu et al., "Controlled attenuation parameter for the detection of steatosis severity in chronic liver disease: a meta-analysis of diagnostic accuracy," Journal of Gastroenterology and Hepatology, vol. 29, no. 6, pp. 11491158, 2014.

[48] Y. Wang, Q. Fan, T. Wang, J. Wen, H. Wang, and T. Zhang, "Controlled attenuation parameter for assessment of hepatic steatosis grades: a diagnostic meta-analysis," International Journal of Clinical and Experimental Medicine, vol. 8, no. 10, pp. 17654-17663, 2015.

[49] K. Pu, Y. Wang, S. Bai et al., "Diagnostic accuracy of controlled attenuation parameter (CAP) as a non-invasive test for steatosis in suspected non-alcoholic fatty liver disease: a systematic review and meta-analysis," BMC Gastroenterology, vol. 19, no. 1, p. 51, 2019.

[50] V. W.-S. Wong, J. Vergniol, G. L.-H. Wong et al., "Liver stiffness measurement using XL probe in patients with nonalcoholic fatty liver disease," American Journal of Gastroenterology, vol. 107, no. 12, pp. 1862-1871, 2012.

[51] V. W.-S. Wong, J. Vergniol, G. L.-H. Wong et al., "Diagnosis of fibrosis and cirrhosis using liver stiffness measurement in nonalcoholic fatty liver disease," Hepatology, vol. 51, no. 2, pp. 454-462, 2010.

[52] R. Kwok, Y.-K. Tse, G. L.-H. Wong et al., "Systematic review with meta-analysis: non-invasive assessment of non-alcoholic fatty liver disease-the role of transient elastography and plasma cytokeratin-18 fragments," Alimentary Pharmacology \& Therapeutics, vol. 39, no. 3, pp. 254-269, 2014. 
[53] J.-F. Cadranel and J.-B. Nousbaum, "Indications de la ponction biopsie hépatique au cours des maladies parenchymateuses diffuses du foie," La Presse Médicale, vol. 41, no. 11, pp. 1064-1070, 2012. 